\title{
A New Research Approach on the Application of Virtual Reality Technology in Civil Engineering

\author{
Changchang $\mathrm{Fu}$
}

Department of Civil Engineering, Zhejiang University of Technology, Hangzhou, 310032, China

\author{
Keywords: Virtual Reality, Civil Engineering, 3D Modeling
}

\begin{abstract}
Virtual reality technology is constantly developing, dedicated to computer graphics and multimedia information processing, high-performance chip can increase a hundred times the processing power, three-dimensional graphics algorithms and parametric modeling algorithm enables virtual reality technology is more mature. This paper, the application of virtual reality technology in civil engineering were studied, the results from the demonstration and validation, planning and design, engineering, construction technology and engineering safety management are discussed in this paper.
\end{abstract}

\section{Introduction}

With the steady growth of China's economy and infrastructure added scale, the scale of construction projects is growing, increasingly complex structure, scientific management of civil engineering, precision increasingly demanding. Realization of civil engineering, information technology, intelligence, visualization and integration become civil construction project management demands of modernization and research focus in this field [1]. Virtual Reality (Virtual Reality, VR) is a comprehensive and highly integrated high-tech, in many areas the military, medicine, design, art, entertainment and other fields have been widely used. Civil Engineering Virtual reality technology in various disciplines involved in civil engineering, has demonstrated some practical, technical potential is huge, very broad application prospects [2-3].

In civil engineering, a long time people had to use abstract concepts represent very rich content, such as using plans, sections, elevations, floor plan and other provisions the formation of some symbols to represent the three dimensional architecture, with more abstract graphics and concise The language used to describe complex scenarios to deliver a lot of information. But this kind of information processing and delivery methods affected employment, knowledge and understanding of the structure of the recipients are engaged in information, communication is very difficult. VR technology development for us to overcome this difficulty provides an extremely effective means. Virtual reality both represent the real world, it can also represent a virtual world.

\section{Virtual Reality Technology Overview}

Virtual reality (VR) a blend of digital image processing, computer graphics, multimedia technology, sensor technology and other information technology branch, greatly promoted the development of computer technology. VR technology is abstract, complex computer data space into an intuitive, user-familiar things. Its essence is to provide an advanced technology of man-machine interface. It is in an analog mode for the user to create a real-time interaction with physical objects reflect changes in the three-dimensional image of the world, in lifelike experience vision, hearing, touch, smell and other acts of perception, so that the participants can directly participate and explore virtual objects and changes in the role of the environment, like being in the real world [4-5]. The architecture is shown in figure 1. 


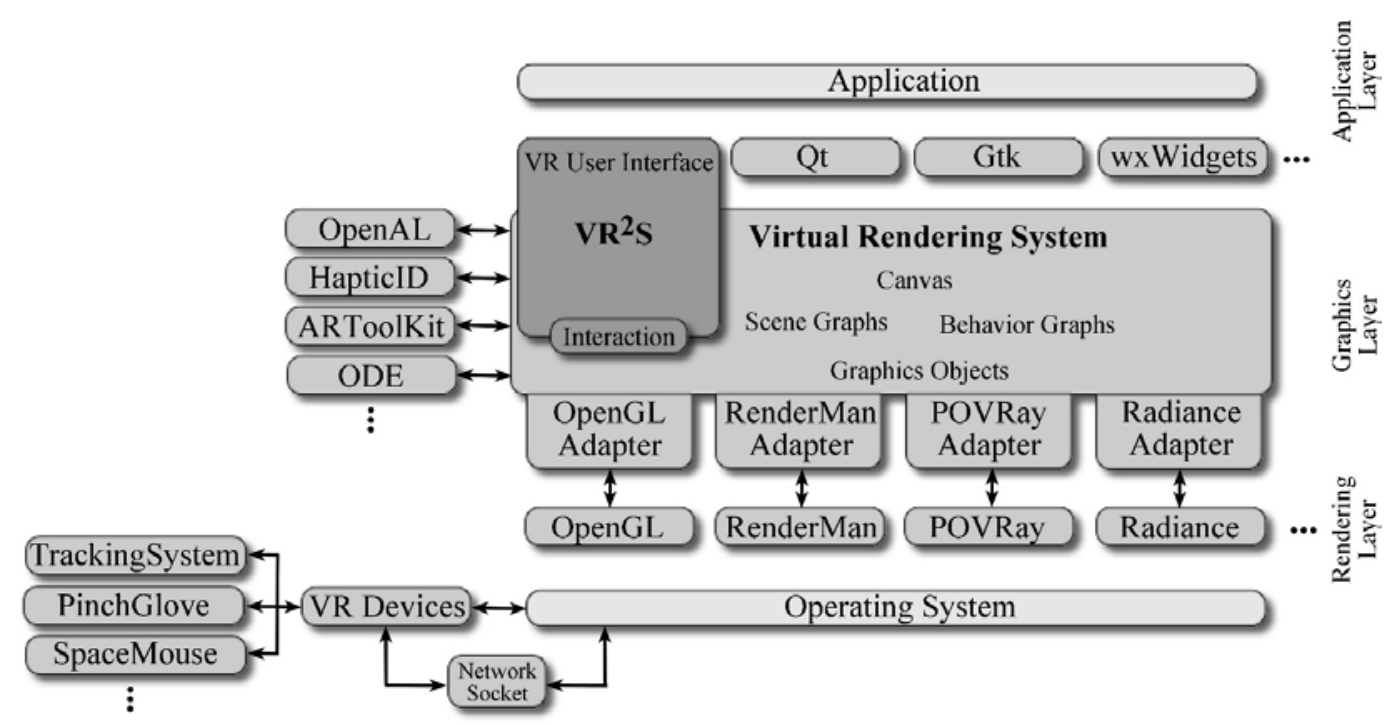

Figure 1.Generic virtual reality software systems architecture

Virtual reality technology has the characteristics of the following three aspects. Is sex. Virtual reality technology is based on human vision, hearing the physiological and psychological characteristics, lifelike three-dimensional image generated by the computer, users wear the helmet mounted display and data gloves and other interactive devices, and can be yourself in a virtual environment, become a member of the virtual environment. The user interaction with various objects in the virtual environment, like in the real world, all feeling is so real, have a feeling of intimacy. Interactivity. Human-computer interaction in virtual reality system is a kind of close to natural interaction, users not only can use the computer keyboard, mouse to interact, but also through the special helmet, such as data glove sensing devices to interact. Users through their natural skills such as language, body movement or action, can for inspection or operation of objects in the virtual environment. More perceptual. Due to see, hear, touch, are installed in the virtual reality system, and kinesthetic

\section{The virtual reality on civil engineering design based on 3D modeling}

Virtual reality on civil engineering based on 3D modeling technology using geometric design, geometric virtual reality technology, is through the 3D and 3D object model scene, usually with the help of a professional modeling software (3ds Max, Maya, etc.) to complete civil engineering design approach that more practical performance scenes and objects in the real world [6], but also to create animations. The effect of virtual reality display rich, powerful, and the interactive is strong.

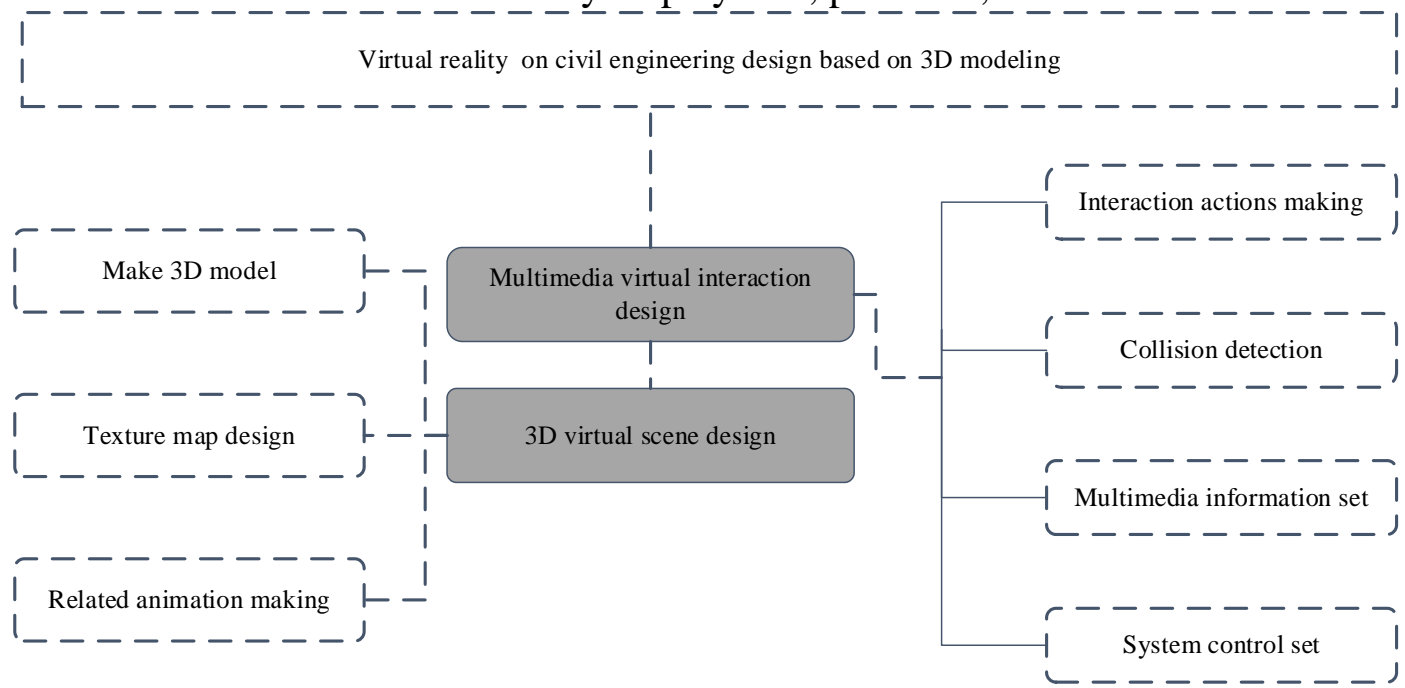

Figure 2. The phase of virtual reality on civil engineering design based on $3 \overline{\mathrm{D}}$ modeling 
The design principle is based on the requirement that through various modeling software, and geometric entities to build a virtual environment, if it satisfies the requirements of virtual reality technology, map rendering, the current mainstream of $3 \mathrm{~d}$ modeling software 3ds Max and Maya, and so can the scene and object modeling, texturing and rendering. After you import the virtual reality software interoperate, multimedia information integration applications and output. Overall, the civil engineering and design based on the principle of virtual reality technology and the main link, shown in Figure 2.

3D virtual reality modeling and civil engineering design, the realization of multiple links design and production needs, from design to the specific process of implementation and suddenly, usually has the following steps:

1) Three-dimensional modeling: designers to design demonstration purposes, the use of advanced CAD, 3 ds Max, Maya, VRML modeling software design and other aspects of digital models, and related optimization.

(2) Structure: three-dimensional model to design a good texture material or map production, lighting and set the scene, adjust the parameters according to different needs.

(3) Anime: Let corresponding animation camera and set the scene.

(4) Interactive: completion of the design, import virtual reality software for editing and realize tiles, add the multimedia information.

(5) Output version: After the virtual design work is completed, select the type of output released into practical use.

\section{Application of virtual reality technology in civil engineering}

Virtual reality technology has been widely used in civil engineering, and has a broad application prospects. This paper mainly from the results of demonstration and validation, planning and design, construction technology and project management, and other aspects of security on virtual reality technology in civil engineering.

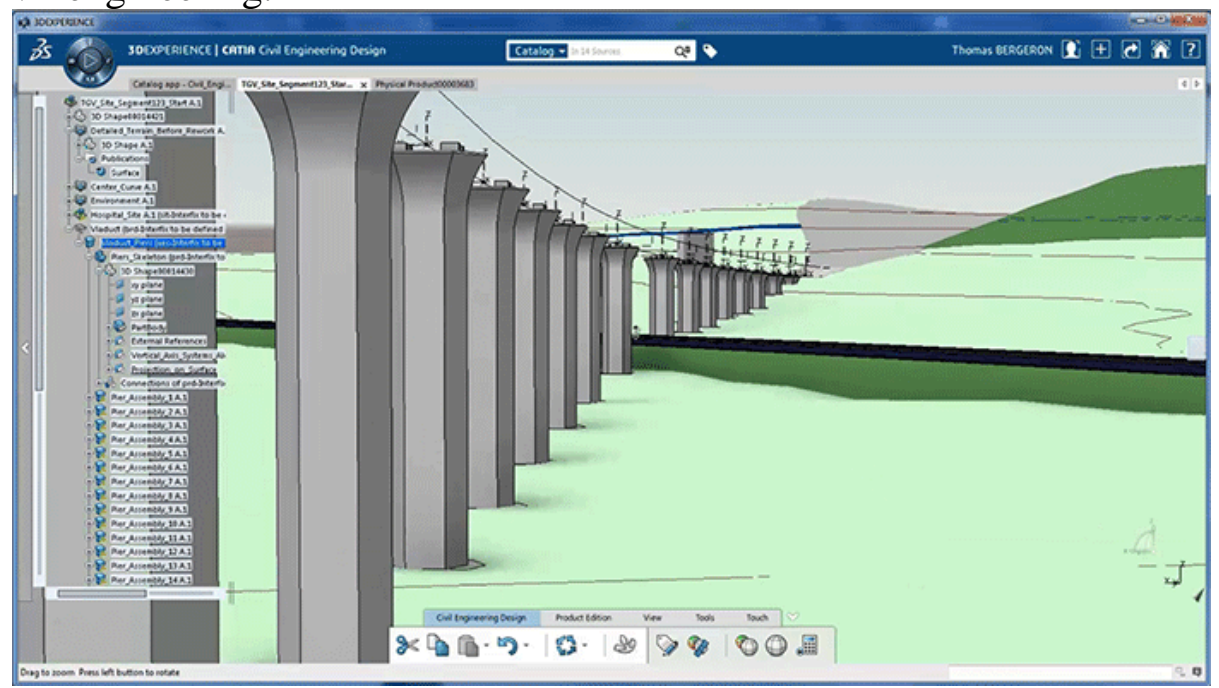

Figure 3.The application case of virtual reality technology in civil engineering

Achievements demonstration and validation. Large-scale public construction projects or important buildings, urban planning, urban transportation planning and design and so requires upfront demonstration, validation. Using virtual reality technology upfront presentation and demonstration, to the real point of view can simulate even any point of view preview of new technologies, to avoid the risk and improve the success rate, but also saves unnecessary waste. Be designed to represent the level of development of modern science and technology building structures, such as ultra-high-rise and large span buildings, large-span bridges, usually to conduct wind tunnel tests, large dams to conduct physical experiments, it is important to carry out destructive experiments structure and then line design, and so on. 
Planning and design. One of the applications of virtual reality technology in architectural design is the virtual reality technology to integrated CAD system, to achieve the desired integration of graphics and structure calculation. Architects use virtual reality CAD system can easily create and modify construction program; then, architects can use already built model, selected structural adjustment program loads and loads defined automatically by the system for structural analysis, the results of , such as the amount of reinforcement and reinforcement diagrams.

Construction Technology and Engineering Safety Management. VR technology using data collected in the field directly intended deflection composite image and design, cable tension, the main beam internal force, stress distribution of dangerous sections, to compare the main beam centroid deflection curve, which does not match the actual parameters of the amendments, to give the construction of rehearsal, can achieve the purpose of construction control. The use of safety engineering VR system that can simulate the process and the possible consequences of accidents occurred in the virtual reality environment. According to the simulation results, the construction side can take appropriate measures to prevent and stop these incidents.

\section{Conclusion}

Virtual reality technology is a large comprehensive integrated environment, is a strong integration of high-tech information technology, because people's participation and control, constitute the man-machine virtual practice, creation and technology has huge potential, money also is big, but it has very broad application prospect. This paper, the application of virtual reality technology in civil engineering were studied, the results from the demonstration and validation, planning and design, engineering, construction technology and engineering safety management are discussed in this paper. Virtual reality technology, however, there are many drawbacks, especially in the study of civil engineering, we should strive to build a virtual reality technology laboratory, geared to the needs of engineering, the development of valuable engineering system, virtual reality in the engineering design, construction, management and visual computing get more extensive application.

\section{Reference:}

[1] Ohta, Yuichi, and Hideyuki Tamura. Mixed reality: Merging real and virtual worlds. Springer Publishing Company, Incorporated, 2014.

[2] Meggs, Susan Martin, Annette Greer, and Sharon Collins. "Virtual reality in interior design education: Enhanced outcomes through constructivist engagement in Second Life." International Journal of Web-Based Learning and Teaching Technologies (IJWLTT) 7.1 (2012): 19-35.

[3] Chi, Hung-Lin, Shih-Chung Kang, and Xiangyu Wang. "Research trends and opportunities of augmented reality applications in architecture, engineering, and construction." Automation in construction 33 (2013): 116-122.

[4] Sampaio, Alcínia Z., and Octávio P. Martins. "The application of virtual reality technology in the construction of bridge: The cantilever and incremental launching methods." Automation in Construction 37 (2014): 58-67.

[5] Sampaio, Alcinia Z., et al. "Virtual reality applied on civil engineering education: Construction activity supported on interactive models." International Journal of Engineering Education 29.6 (2013): 1331-1347.

[6] Cheng, Tao, and Jochen Teizer. "Real-time resource location data collection and visualization technology for construction safety and activity monitoring applications." Automation in Construction 34 (2013): 3-15. 